\title{
On Scheduling and Interference Coordination Policies for Multicell OFDMA Networks
}

\author{
Gábor Fodor \\ Ericsson Research, Kista, Sweden \\ Gabor. Fodoreericsson. com
}

\begin{abstract}
In orthogonal frequency division multiple access systems there is an intimate relationship between the packet scheduler and the inter-cell interference coordination (ICIC) functionalities: they determine the set of frequency channels (sub-carriers) that are used to carry the packets of in-progress sessions. In this paper we build on previous work - in which we compared the so called random and coordinated ICIC policies - and analyze three packet scheduling methods. The performance measures of interest are the session blocking probabilities and the overall throughput. We find that the performance of the so-called Fifty-Fifty and What-It-Wants scheduling policies is improved by coordinated sub-carrier allocation, especially in poor signal-to-noise-and-interference situations. The performance of the All-Or-Nothing scheduler is practically insensitive to the choice of the sub-carrier allocation policy.
\end{abstract}

Keywords: Orthogonal Frequency Division Multiple Access, Radio Resource Management, Interference Coordination, Scheduling.

\section{Introduction}

The $3^{\text {rd }}$ Generation Partnership Project (3GPP) has selected orthogonal frequency division multiple access (OFDMA) as the radio access scheme for the evolving universal terrestrial radio access (E-UTRA). Packet scheduling (PSC) and inter-cell interference coordination (ICIC) are important radio resource management (RRM) techniques that together determine the set of OFDMA resource blocks (essentially the sub-carriers) that are taken into use when a packet is scheduled for transmission over the radio interface [2], [3]. In broad terms, PSC is responsible for determining the session(s) that can send a packet during a scheduling interval and the number of sub-carriers that the session may use. The number of the assigned sub-carriers has a direct impact on the instantaneous bit-rate and thereby can be seen as part of the rate control mechanism. The ICIC function, in turn, is concerned with allocating the particular sub-carriers to the session taking into account the instantaneous channel conditions and the ICIC policy. Such ICIC policy may coordinate which sub-carriers should be taken into use by the schedulers in neighbor cells.

The impact of these two RRM functions on the session-wise and overall throughput has been for long recognized by the standardization and research communities. Sections 11.2.4 and 11.2.5 of [2] and Chapter 6.12 of [3] describe the roles of the PSC and ICIC functions and discuss their relation. From a performance analysis perspective, 
Letaief et al. developed a model that jointly optimizes the bit and power allocation in OFDMA schedulers [5]. ICIC has been the topic of research for long (for a classical overview paper, see [4]). The paper by Liu and Li proposed a so called "Radio Network Controller algorithm" that determines the set of allowed resources in each base station under its control, while the "Base Station algorithm" schedules packets for transmission [9] (see also Chapter 8 of [10]). These works demonstrate that already with a single dominant interfering neighbor cell, the total throughput increases when an appropriate ICIC policy is employed by the packet scheduler.

The contribution of the current paper is that we (1) explicitly take into account that traffic is elastic and (2) propose a flexible model to capture the behavior of a wide range of schedulers under two different ICIC policies. With regards to (1) we allow the bitrates of the sessions to fluctuate between the associated minimum and maximum rates. This model allows the maximum rate to be large so that the behavior of TCP-like greedy sources can be captured. Regarding (2), we introduce the notion of the scheduler policy vector that specifies the probability that a session is granted a certain amount of subcarriers when there are competing sessions in the system. We add this rather general scheduler model to the interference coordination model described in [8] and analyze the model in a sequence of steps (Steps 1-6) detailed in the paper. The performance analysis gives insight into the potential gains that inter-cell interference coordination can give when employing different packet scheduling policies.

The paper is organized as follows. In the next section, we describe the scheduling and ICIC policies that we study and introduce the policy vector as a convenient tool to characterize these policies. Next, in Section 3 we state the performance analysis objective in terms of the input parameters and the performance measures of interest. The solution is summarized in a sequence of steps (as described above). Section 4 discusses numerical results. We highlight our findings in Section 5. We note that the proofs of the lemmas as well as further details, numerical results and the conclusions are available in the longer version of the paper [1].

\section{Scheduling and Inter-cell Interference Coordination Policies}

We consider an OFDMA cell that comprises $S$ orthogonal frequency channels (subcarriers). The number of in-progress sessions is denoted by $i$ and represents the state of the system. When the system is in state $i$, the scheduler determines the number of sub-carriers that are assigned to each session. For a particular session under study, this implies that the session is assigned $s$ number of sub-carriers with probability $P(s)$; $\sum_{s=0}^{S} P(s)=1$. We refer to the mechanism that (in each system state) establishes $P(s)$ as the scheduling policy. The scheduling policy vector is a vector of dimension $(S+1)$ whose $s^{t h}$ element specifies the probability that the session under study (and thereby any session) is allocated $s$ channels, $s=0 \ldots S$. (We note that the indexing of the $(S+1)$ elements of the policy vector runs from 0 to $S$.) In the following subsections we describe three such scheduling polices.

Throughout we assume that the sessions belong to the same service class that is characterized by a peak rate requirement $\hat{R}$ and a maximum slowdown factor $\hat{a} \geq 1$. The minimum accepted (guaranteed) bit rate for a session is $R_{\text {min }}=\hat{R} / \hat{a}$. Also, when 
a session is granted $s$ number of frequency channels, its ideal bit-rate (assuming a given and fixed modulation and coding scheme, MCS) and assuming zero packet error/loss rate $(P E R=0)$ is denoted by $R_{s}$. When $\hat{R}$ is set to $R_{S}$ (that is the peak bit-rate requirement is the bit-rate that is provided when all resources are assigned to a single session), we say that the session is greedy. We will also use the operator $\mathcal{S}(R)$ that returns the number of required channels in order for the session to experience $R$ bitrate (again assuming $P E R=0$ ). That is, when a session is admitted into the system, the number of allocated channels $s$ (in the long term) must fulfil: $R_{\min } \leq R_{s} \leq \hat{R}$. This implies that we assume that an admission control procedure operates in the system such that the maximum number of simultaneously admitted sessions remain under $\hat{I} \triangleq$ $\left\lfloor\frac{S}{\mathcal{S}(\hat{R} / \hat{a})}\right\rfloor$. We say that state $i$ is an under-loaded, critically loaded or overloaded state if $\mathcal{S}(i \cdot \hat{R})$ is less than, equal to or greater than $S$ respectively.

\subsection{The What-It-Wants Scheduling Policy}

The What-It-Wants scheduling policy attempts to grant $\mathcal{S}(\hat{R})$ channels to the sessions as long as $i \cdot \mathcal{S}(\hat{R}) \leq S ; \quad i>0$. Otherwise, in overloaded states, it grants either $\left\lfloor\frac{S}{i}\right\rfloor$ or $\left\lceil\frac{S}{i}\right\rceil$ channels. Specifically, the What-It-Wants scheduling policy is defined by the following Policy Vector. If $i \cdot \mathcal{S}(\hat{R}) \leq S$ :

$$
\vec{P}_{W I W}(s)=\left\{\begin{array}{l}
1 \text { if } s=\mathcal{S}(\hat{R}) \\
\quad \text { granting peak rate with prob. 1), } \\
0 \text { otherwise. }
\end{array}\right.
$$

For overloaded states, we need to distinguish between two cases. If $\frac{S}{i}$ is an integer number, then:

$$
\vec{P}_{W I W}(s)=\left\{\begin{array}{l}
1 \text { if } s=\frac{S}{i} \\
\quad \text { granting an equal share with prob. 1) } \\
0 \text { otherwise. }
\end{array}\right.
$$

When $\frac{S}{i}$ is not an integer number, the following relations must hold. The scheduler grants $\left\lfloor\frac{S}{i}\right\rfloor$ channels with probability $P_{1}$ and $\left\lceil\frac{S}{i}\right\rceil$ number of channels with probability $1-P_{1}$. Clearly:

$$
P_{1} \cdot\left\lfloor\frac{S}{i}\right\rfloor+\left(1-P_{1}\right) \cdot\left\lceil\frac{S}{i}\right\rceil=\frac{S}{i} ; \quad P_{1}=\left\lceil\frac{S}{i}\right\rceil-\frac{S}{i} .
$$

Thus, the policy vector in this case takes the form:

$$
\vec{P}_{W I W}(s)= \begin{cases}P_{1} & \text { if } s=\left\lfloor\frac{S}{i}\right\rfloor \\ 1-P_{1} & \text { if } s=\left\lceil\frac{S}{i}\right\rceil \\ 0 & \text { otherwise. }\end{cases}
$$




\subsection{The All-Or-Nothing Scheduling Policy}

In the All-Or-Nothing scheduling policy all resources are assigned to the scheduled session. This type of scheduling is employed in High Speed Downlink Packet Access (HSDPA) systems when code multiplexing is not used. Thus, a session with peak rate requirement $\hat{R}$ would need to be scheduled with probability $\mathcal{S}(\hat{R}) / S$ in order for it to receive its peak rate. However, when there are $i \geq 1$ on-going sessions, any given session cannot get scheduled with higher probability than $1 / i$. That is, in the All-OrNothing scheduling policy, in system state $i$, a session gets scheduled with probability $P_{2}=\operatorname{Min}[\mathcal{S}(\hat{R}) / S, 1 / i]$. The scheduling policy takes the following form:

$$
\vec{P}_{A o N}(s)= \begin{cases}P_{2} & \text { if } s=S \\ 1-P_{2} & \text { if } s=0 \\ 0 & \text { otherwise. }\end{cases}
$$

\subsection{The Fifty-Fifty Scheduling Policy}

The Fifty-Fifty scheduling policy can be seen as a policy in between the What-It-Wants and All-Or-Nothing policies. When there are $i$ sessions in the system, the scheduler divides the resources (almost) equally between the competing sessions (similarly to WhatIt-Wants). However, similarly to the All-Or-Nothing policy, in under-loaded states this would mean that the sessions receive more resources in the long term than $\mathcal{S}(\hat{R})$. Thus, in this policy, in underloaded state $i$, if $\frac{S}{i}$ is not integer, a session receives $\left\lfloor\frac{S}{i}\right\rfloor$ channels with probability $P_{31},\left\lceil\frac{S}{i}\right\rceil$ number of channels with probability $P_{32}$ and no channels with probability $1-P_{31}-P_{32}$. Clearly, in states for which $i \cdot \mathcal{S}(\hat{R})<S$ and $\frac{S}{i}$ is not an integer number:

$$
P_{31} \cdot\left\lfloor\frac{S}{i}\right\rfloor+P_{32} \cdot\left\lceil\frac{S}{i}\right\rceil=\mathcal{S}(\hat{R}), \text { and } P_{31}: P_{32}=\left(\left\lceil\frac{S}{i}\right\rceil-\frac{S}{i}\right):\left(\frac{S}{i}-\left\lfloor\frac{S}{i}\right\rfloor\right) .
$$

If $\frac{S}{i}$ is integer, the session is assigned $\frac{S}{i}$ number of channels with probability $P_{33}$ and zero channels with probability $1-P_{33}$ :

$$
P_{33} \cdot \frac{S}{i}=\mathcal{S}(\hat{R}) ; \quad \text { and: } \quad P_{0}=1-P_{33} .
$$

For critically and overloaded states $(i \cdot \mathcal{S}(\hat{R}) \geq S)$ the channels are fully utilized $\left(P_{34}+P_{35}=1\right)$ :

$$
P_{34} \cdot i \cdot\left\lfloor\frac{S}{i}\right\rfloor+P_{35} \cdot i \cdot\left\lceil\frac{S}{i}\right\rceil=S .
$$

In the critically loaded and overloaded states, if $\frac{S}{i}$ is integer, the number of allocated sessions for each session is $\frac{S}{i}$ with probability 1 . Based on these observations, the scheduling policy vector for the Fifty-Fifty policy is straightforward to determine (although a bit tedious to formally specify it, see [1]. 

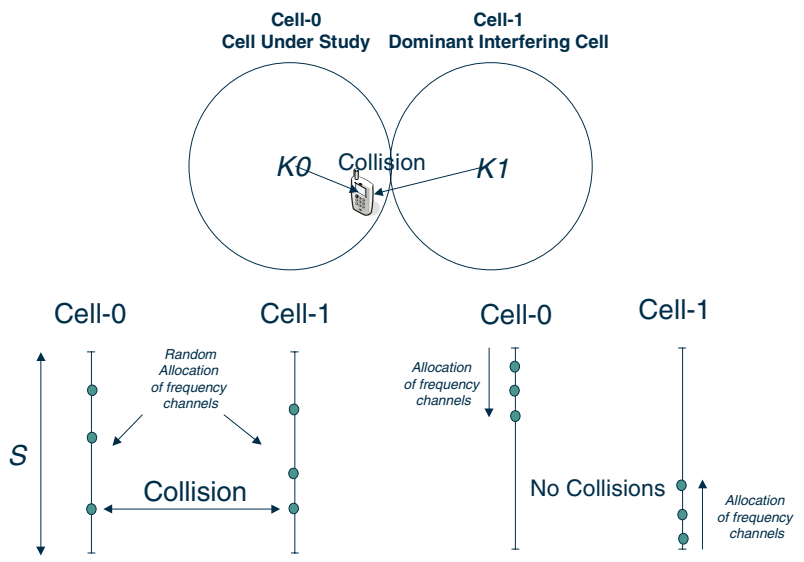

Fig. 1. Random and Coordinated ICIC policies. Coordinated ICIC can be realized by assigning a cell specific ordered list of the frequency channels to each cell such that the "collisions" of frequency channels are avoided as long as there are non-colliding pairs. Assuming a single (dominant) interfering cell (as in [9] and [10]), devising such ordered lists is straightforward. For many cells, coordinated ICIC implies careful frequency planning, as described in for example [4].

\subsection{A Numerical Example}

Consider an OFDMA cell that supports $S=64$ sub-carriers (channels). Sessions have a peak rate requirement that corresponds to $\mathcal{S}(\hat{R})=4$ channels. When there are 6 inprogress sessions, the system is under-loaded $(6 \cdot 4<64)$, the three scheduling policy vectors are as follows:

$$
\begin{aligned}
\vec{P}_{W I W} & =[0,0,0,0,1,0 \ldots, 0] \\
\vec{P}_{A o N} & =\left[\frac{60}{64}, 0, \ldots, 0, \frac{4}{64}\right] ; \\
P_{F F} & =\left[\frac{40}{64}, 0, \ldots, 0, \frac{8}{64}, \frac{16}{64}, 0, \ldots, 0\right],
\end{aligned}
$$

where the $P_{F F}$ vector has non-zero elements at positions 0, 10 and 11 (corresponding to 1,11 and 12 scheduled channels). Since the system is underloaded, the What-It-Wants policy grants the peak rate with probability 1 (4 channels), the All-Or-Nothing policy allocates all the 64 channels with probability 4/64. The Fifty-Fifty policy $(A=0.0625$, $B=0.03125$ so $P_{31}=0.125$ and $P_{32}=0.25$ ) either allocates 10 or 11 channels to any given session (with probabilities 8/64 and 16/64 respectively) or it does not schedule the session (zero channels with probability 40/64). (All three policies allocate 4 channels in the long term average in this system state.)

\subsection{ICIC Policies: Random and Coordinated Sub-Carrier (Channel) Allocation}

Basically, there are two approaches as to how the sub-carriers out of the available ones are selected when a session requires a certain number of sub-carriers (see Figure1). The 
simplest way is to pick sub-carriers out of the ones that are available (i.e. scheduled) randomly such that any available sub-carrier has the same probability to get allocated to an arriving session. Random allocation of sub-carriers is attractive, because it does not require any coordination between cells, but it may cause collisions even when there are free sub-carriers. In contrast, a low complexity coordination can avoid collisions as long as there are non-colliding sub-carrier pairs in the two-cell case and non-colliding tuples in the multiple-cell case. We refer to this method as coordinated sub-carrier allocation. (Further details about these ICIC policies in general can be found in [8].)

\section{Performance Measures of Interest and Solution Approach}

\subsection{Input Parameters and Performance Measures}

We consider a single OFDMA cell with $S$ channels at which sessions belonging to the same (elastic) service class arrive according to a Poisson process of intensity $\lambda$. Each session brings with itself a file whose size is an exponentially distributed random variable with parameter $\mu$. The session requests a radio bearer that is characterized by its peak rate $\hat{R}$ (for which: $\mathcal{S}(\hat{R}) \leq S$ ) and minimum rate $\hat{R} / \hat{a}$, where $\hat{a} \geq 1$ is the maximum slowdown factor associated with the session. If, at the time instant of the arrival of the new session, the admission of the new session brought the system into a state in which the minimum rate (governed by the particular scheduling policy) cannot be granted, the session is blocked and leaves the system. The single cell is disturbed (interfered) by a single dominant interferer cell, such as in [9]. In this paper we characterize the load in this dominant interfering cell by the number of used sub-carriers $K_{1} \leq S$. When an allocated sub-carrier in the cell under study and one of the $K_{1}$ disturbing channels use the same sub-carrier frequency, we say that the two sub-carriers collide and suffer from co-channel interference [4].

The performance measures of interest are the session-wise blocking probability and the mean file transfer time. These two quantities represent a trade-off since more admitted sessions imply lower per-session throughput and thereby longer file transfer times. This trade-off in a WCDMA environment has been investigated by Altman in [6] and subsequently by Fodor et al. in [7].

\subsection{Step 1: Determining the Distribution of the Allocated Sub-Carriers}

Recall that in each system state the scheduling policy vector determines the probability that a given session is allocated $s$ channels. When a session is given $s$ channels (which happens with probability $\vec{P}(s)$ ), we need to calculate the conditional distribution of the number of the totally allocated number of channels (that is to all sessions) in the cell (denoted by $K_{0}$ ), given that the session under study is given $s$ channels. This is because $K_{0}$ and the number of disturbing channels $K_{1}$ determine the distribution of the colliding and collision-free channels in the cell, which in turn determine the performance measures of interest.

We cannot give a closed form formula for the (conditional) distribution of $K_{0}$. However, in [1] we provide the pseudo code description of the algorithm that calculates it. 


\subsection{Step 2: Determining the Distribution of the Colliding Sub-Carriers Under the Random and Coordinated Sub-Carrier Allocation Policies}

Lemma 1. Let $S$ denote the total number of available sub-carriers in each cell and let $K_{0} \leq S$ and $K_{1} \leq S$ denote the number of allocated channels in Cell-0 and Cell-1 respectively. Let $N_{1}(c)$ denote the number of possible channel allocations in Cell-O and Cell-1 such that the number of collisions is $c$.

Then, the distribution and the mean of the number of collisions under the random allocation policy $\left(\gamma_{1}\right)$ are as follows:

$$
\begin{gathered}
c_{M I N}=\operatorname{Max}\left[0, K_{0}+K_{1}-S\right], \quad c_{M A X}=\operatorname{Min}\left[K_{0}, K_{1}\right], \\
N_{1}(c)=\left(\begin{array}{c}
S \\
c
\end{array}\right) \cdot\left(\begin{array}{c}
S-c \\
K_{0}-c
\end{array}\right) \cdot\left(\begin{array}{c}
S-K_{0} \\
K_{1}-c
\end{array}\right) ; \quad \operatorname{Pr}\left\{\gamma_{1}=c \mid K_{0}, K_{1}\right\}=\frac{N_{1}(c)}{T O T 1}, \\
E\left[\gamma_{1} \mid K_{0}, K_{1}\right]=\sum_{c=c_{M I N}}^{c_{M A X}} \frac{c \cdot N_{1}(c)}{T O T 1}, \quad \text { where TOT1 }=\left(\begin{array}{c}
S \\
K_{0}
\end{array}\right) \cdot\left(\begin{array}{c}
S \\
K_{1}
\end{array}\right) .
\end{gathered}
$$

Lemma 2. Using similar notation as in Lemma 1 the distribution and the mean number of collisions under the coordinated allocation policy $\left(\gamma_{2}\right)$ is given by:

$$
\begin{gathered}
N_{2}(c)= \begin{cases}1 & \text { if } c=c_{0} \\
0 & \text { otherwise, }\end{cases} \\
c_{0}=\left\{\begin{array}{l}
0 \\
K_{0}+K_{1}-S \quad \text { if } K_{0}+K_{1}<S,
\end{array}\right. \\
\operatorname{Pr}\left\{\gamma_{2}=c\right\}=N_{2}(c), \quad E\left[\gamma_{2}\right]=\sum_{c=c_{M I N}}^{c_{M A X}} c \cdot N_{2}(c) .
\end{gathered}
$$

\subsection{Step 3: Determining the Packet-Wise Effective SINR}

The scheduling policy vector specifies the probability that $s$ channels are used in Cell-0, whereas Lemmas 1-2 determine the probability that the number of colliding channels is $c$. We will use the following lemma to determine the probability that the number of colliding channels in a packet of size $L$ is $\gamma$ when the number of scheduled channels (for the session under study) is $s$ and the total number of colliding channels is $c \leq s$.

\section{Lemma 3}

$$
\operatorname{Pr}\{\gamma \leq c \text { channels out of } L \text { are colliding }\}=\left(\begin{array}{l}
L \\
\gamma
\end{array}\right) \cdot \frac{\left(\begin{array}{l}
c \\
\gamma
\end{array}\right)}{\left(\begin{array}{l}
s \\
\gamma
\end{array}\right)} \cdot \frac{\left(\begin{array}{l}
s-c \\
L-\gamma
\end{array}\right)}{\left(\begin{array}{c}
s-\gamma \\
L-\gamma
\end{array}\right)}
$$




\subsection{Step 4: Calculating the SINR Level in Case of Collisions for the Downlink}

Lemmas 1-3 determine the probability that the number of colliding channels is $\gamma$ and the number of non-colliding channels is $L-\gamma$ in a packet of a session under study. We now need to determine the impact of the collision on a channel's signal-to-noise-andinterference (SINR) ratio.

For this, we use the path loss model recommended by the 3GPP (described in [15]) and a result from [8]. Let $\theta$ be a predefined threshold and let $X \triangleq \frac{r_{0}}{r_{1}}$ be a random variable representing the distance ratio between the mobile station distances from its serving and disturbing base station respectively. Also, let $Q_{0}$ and $Q_{1}$ denote the power that the serving and the neighbor base station uses on the colliding channels respectively. Furthermore, let $G_{0}$ and $G_{1}$ denote the path gains from the serving base station (that is in Cell-0) and the dominant neighbor base station (that is in Cell-1) respectively to the mobile station under study. Then, the probability that the SINR remains under this threshold can be approximated as follows [8]:

$$
\begin{gathered}
\operatorname{Pr}\left(\frac{G_{0} \cdot Q_{0}}{G_{1} \cdot Q_{1}+N_{0}}<\theta\right) \approx \int_{0}^{\operatorname{Max}[X]}\left(f_{X}(x) g(x)\right) d x \\
g(x) \triangleq \frac{1}{2} \operatorname{erfc}\left(-\frac{5}{b \varsigma} \cdot \frac{\ln \frac{x^{\mu} \theta}{Q_{0} / Q_{1}}}{\ln 10}\right) .
\end{gathered}
$$

where $f_{X}(x)$ is the probability density function of $X ; b, \varsigma$ and $\mu$ are the parameters of the 3 GPP path loss model as described in [15].

\subsection{Step 5: Calculating the Effective SINR and the Packet Loss Probability}

We are now in the position that the packet loss probability in each system state can be determined.

When one or more of the channels that are used to carry a packet are hit by collisions, an efficient way to characterize the overall SINR quality of the packet is to use the notion of the effective SINR. This concept has been proposed in [12] and used in for instance [13], in which a method to calculate the packet error probability for a given value of the effective SINR was also proposed. A specific method to calculate the effective SINR (based on the SINR of the composing channels) that is applicable in cellular OFDM systems is also recommended by the 3GPP [11].

In this paper we employ the 3 GPP method that can be summarized as follows. Suppose that there are $L$ sub-carriers that carry a data packet and each has a SINR value of $S I N R_{i}$. Then, the effective SINR that is assigned to the packet is given by:

$$
S I N R_{\mathrm{eff}}=\alpha_{1} \cdot I^{-1}\left(\frac{1}{L} \sum_{i=1}^{L} I\left(\frac{S I N R_{i}}{\alpha_{2}}\right)\right),
$$

where $I(\cdot)$ is a model specific function and $I^{-1}(\cdot)$ is its inverse. The parameters $\alpha_{1}$ and $\alpha_{2}$ allow to adapt the model to characteristics of the considered modulation and coding scheme. The exponential effective SINR metric proposed in [11] corresponds 
to $I(x)=\exp (-x)$. In [13] it is shown that for QPSK and 16-QAM modulation, the parameters $\alpha_{1}$ and $\alpha_{2}$ can be chosen as follows: $\alpha_{1}=1$ and $\alpha_{2}=1$. In [13] a method to determine the packet error rate $(\sigma)$ as a function of the effective SINR is presented. Essentially, this method maps (in a 1-1 fashion) the effective SINR onto a (modulation and coding scheme dependent) packet error rate.

\subsection{Step 6: Determining the Performance Measures of Interest}

We now make use of the assumption that the session arrivals form a Poisson process and that the session size is exponentially distributed. We choose the number of admitted sessions as the state variable and thus the number of states in the system is $\hat{I}+1$. The transitions between states are due to an arrival or a departure of a session. The arrival rates are given by the intensity of the Poisson arrival processes. Due to the memoryless property of the exponential distribution, the departure rate from each state depend on the nominal holding time of the in-progress sessions, and also on the slow down factor and the packet error rate in that state. Specifically, when the slow down factor is $a_{i}(n)$, and the packet error rate is $\sigma(n)$ its departure rate is $\left(1-\sigma_{i}(n)\right) \mu_{i} / a_{i}(n)$.

The Markovian property for such systems was observed and formally proven by Altman et al. in [14]. Thus, the system under these assumptions is a continuous time Markov chain whose state is uniquely characterized by the state variable $n$.

\section{Numerical Results}

In accordance with the 3GPP recommendation, we here (in a somewhat simplified fashion) assume that a downlink resource block (sometimes referred to as a chunk) occupies $300 \mathrm{kHz}$ and $0.5 \mathrm{~ms}$ in the frequency and time domains respectively. A chunk carries 7 OFDM symbols on each sub-carrier; therefore the downlink symbol rate is $R_{\text {symbol }}=140$ symbols/chunk/0.5ms. Assuming a $10 \mathrm{MHz}$ spectrum band, and considering some overhead due to measurement reference symbols and other reasons, this corresponds to 30 chunks in the frequency domain $(S=30)$, that is $8400 \mathrm{ksymbol} / \mathrm{s}$. The actual bit-rate depends on the applied modulation and coding scheme, in this paper we do not model adaptive modulation and coding (AMC), we simply assume a fixed binary phased shift keying (BPSK) so that each symbol carries $n_{M C S}=2$ bits. Sessions arrive according to a Poisson process of intensity $\lambda=1 / 8[1 / s]$. A session is characterized by the amount of bits that it transmits during its residency time in the system (we may think of this quantity as the size of the file that is to be downloaded). We assume that this file size is an exponentially distributed random variable with mean value $\nu=4 * S * R_{\text {symbol }} * n_{M C S}$. The blocking probability and file download meantime results are shown in Figures 2, 3. On the $x$ axis we let the number of disturbing channels (i.e. the occupied channels in the neighbor cell) increase $(K 1 / 5=1 \ldots 6)$, while the $y$ axis shows the blocking probabilities and the mean session residency times. The upper graphs in each figure correspond to the case when there is no channel allocation coordination between the cells, while the lower graphs assume coordination (channel segregation). This system is highly loaded so that when $\hat{a}$ is set to 1 , the blocking probabilities increase from $5.5 \%$ up to $7 \%$, while the file download time increases from 30.5 $\mathrm{s}$ to $33 \mathrm{~s}$ (not shown here). The figures correspond to the case when $\hat{a}=2$. We observe 

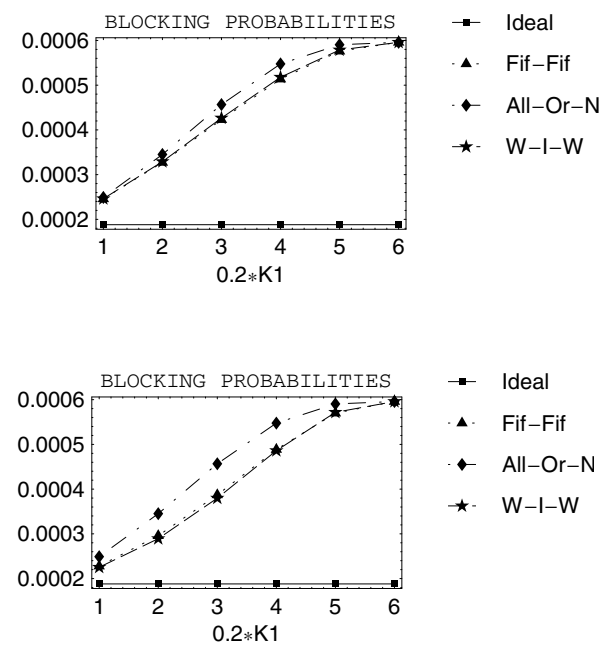

Fig. 2. Blocking probabilities as the function of the number of disturbing channels in the dominant interferer cell $(K 1=5,10,15,20,25,30)$. When the sessions tolerate some slowdown (here $\hat{a}=2$, that is $R_{\min }=\hat{R} / 2$ ), the blocking probabilities are low, (in this example 2 orders of magnitude lower than when $\hat{a}=1$ (not shown here)), and the coordinated allocation (lower diagram) performs somewhat better when the scheduling method is the All-Or-Nothing or What-It-Wants ("W-I-W").
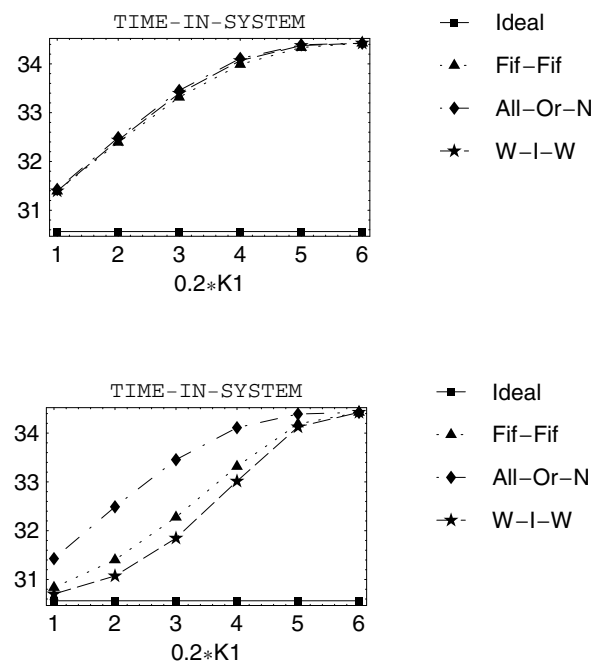

Fig. 3. Average file download time as the function of the number of disturbing channels in the dominant interferer cell $(K 1=5,10,15,20,25,30)$. When the sessions tolerate some slowdown (here $\hat{a}=2$, that is $R_{\text {min }}=\hat{R} / 2$, the session holding time increases somewhat, (as compared to the case when $\hat{a}=1$ (not shown here)), and the coordinated allocation again performs somewhat better when the scheduling method is the All-Or-Nothing or What-It-Wants ("W-I-W"). 
that when the sessions tolerate some slowdown, the blocking probability dramatically decreases (down to $0.06 \%$ !) without much increasing the download time (from around $33 \mathrm{~s}$ to around $34 \mathrm{~s}$ ). Secondly, we note that coordinated allocation is beneficial when the What-It-Wants or the Fifty-Fifty scheduling method is employed, and has no effect when the All-Or-Nothing scheduling is used. The curve denoted "ideal" corresponds to the case when the packet error rate $\sigma$ is zero in all system states. We refer to [1] for further results and analysis.

\section{Conclusion}

Inter-cell interference coordination is an important radio resource management function for (O)FDMA based cellular systems in general [4] and for the evolving Universal Terrestrial Radio Access Network (E-UTRA) in particular [2], [3]. We proposed the notion of the (scheduling) policy vector to model the behavior of the packet scheduler. Using the policy vector, we were able to derive the conditional distribution of the number of colliding and collision free channels in the cell under study for all three cases. This in turn allowed us to determine the distribution of the number of colliding and collision free (i.e. co-channel interference free) channels in each scheduled packet. We used this knowledge to calculate the effective SINR and from it the packet error rate and thereby the useful packet throughput of the system. This useful throughput determines the session wise blocking probabilities and the time it takes for elastic sessions to complete a file transfer.

Our major finding is that the performance of the ICIC function (its impact on the system throughput) depends on the employed scheduler. Specifically, for peak rate limited ("narrow band") traffic (that is when $\mathcal{S}(\hat{R})<S$ ), when frequency domain scheduling is used in combination with time domain scheduling, it is useful to employ coordinated channel allocation in neighbor cells. Coordinated ICIC has little impact when the scheduler is pure time domain based. We also note that our further numerical results indicate that ICIC is only necessary for cell edge users, whose SINR is negatively impacted by frequency domain collisions [1].

\section{References}

1. G. Fodor and M. Telek, "Modeling and Performance Analysis of Scheduling Poicies for OFDMA Based Evolved UTRA", Technical Report, 2006. http://webspn. hit.bme.hu/ telek/techrep/multicell.pdf

2. 3GPP Technical Report TR 25.912, Feasibility Study for Evolved Universal Terrestrial Radio Access (E-UTRA), Release 7, 2006.

3. 3GPP Technical Report TR R3.018, Evolved UTRA and UTRAN Radio Access Architecture and Interfaces, Release 7, 2006.

4. I. Katzela and M. Naghshineh, "Channel Assignment Schemes for Cellular Mobile Telecommunication Systems: A Comprehensive Survey", IEEE Personal Communications, pp. 1031, June 1996.

5. Ying Jun Zhang and Khalid Ben Letaief, "Multiuser Adaptive Subcarrier-and-Bit Allocation With Adaptive Cell Selection for OFDM Systems", IEEE Transactions on Wireless Communications, Vol. 3, No. 5, pp. 1566-1575, September 2004. 
6. E. Altman, "Capacity of Multi-service Cellular Networks with Transmission-Rate Control: A Queueing Analysis", ACM Mobicom '02, Atlanta, GA, September 23-28, 2002.

7. G. Fodor and M. Telek, "Performance Anlysis of the Uplink of a CDMA Cell Supporting Elastic Services", in the Proc. of IFIP Networking 2005, Waterloo, Canada, Springer LNCS 3462, pp. 205-216, 2005.

8. G. Fodor, "Performance Analysis of a Reuse Partitioning Technique for OFDM Based Evolved UTRA", $14^{\text {th }}$ IEEE International Workshop on QoS, New Haven, CT, USA, pp. 112-120, June 2006.

9. G. Li and H. Liu, "Downlink Dynamic Resource Allocation for Multi-cell OFDMA System", $58^{\text {th }}$ IEEE Vehicular Technology Conference, VTC 2003-Fall, Vol. 3, pp. 1698-1702, 6-9 October 2003.

10. H. Li and G. Liu, "OFDM-Based Broadband Wireless Networks: Design and Optimization”, WILEY, 2005, ISBN: 0471723460.

11. 3GPP Technical Report TR 25.892, Feasibility Study for OFDM for UTRAN Enhancements (Release 6), V6.0.0, 2004-06.

12. S. Nanda and K. M. Rege, "Frame Error Rates for Convolutional Codes on Fading Channels and the Concept of Effective $E_{b} / N_{0}$ ", IEEE Transactions on Vehicular Technologies, Vol. 47, No. 4, pp. 1245-1250, November 1998.

13. K. Brueninghaus, D. Astély, T. Sälzer, S. Visuri, A. Alexiou, S. Karger, G-A Seraji, "Link Performance Models for System Level Simulations of Broadband Radio Access Systems", $16^{\text {th }}$ IEEE International Symposium on Personal, Indoor and Mobile Communications, PIMRC, 11-14 Sepetmeber 2005.

14. E. Altman, D. Artiges and K. Traore, "On the Integration of Best-Effort and Guaranteed Performance Services”, INRIA Research Report No. 3222, July, 1997.

15. 3GPP TR 25.942, Radio Frequency System Scenarios, 2005. 